\title{
Are adipokines associated with gestational diabetes mellitus?
}

\author{
Ümit Görkem ${ }^{1}$, Ferit Kerim Küçükler ${ }^{2}$, Cihan Toğrul ${ }^{1}$, Tayfun Güngör ${ }^{1}$ \\ ${ }^{1}$ Department of Obstetrics and Gynecology, Hitit University School of Medicine, Çorum, Turkey \\ ${ }^{2}$ Department of Endocrinology, Hitit University School of Medicine, Çorum, Turkey
}

\begin{abstract}
Objective: To investigate the association of maternal serum levels of chemerin, retinol binding protein-4 (RBP-4), and visfatin with gestational diabetes mellitus (GDM).

Material and Methods: 158 pregnant women were screened between 24 and 28 weeks of gestation. They were divided into two groups: GDM group $(n=76)$ and control group $(n=82)$. Maternal serum concentrations of chemerin, RBP-4, visfatin, insulin, and homeostasis model assessment-insulin resistance (HOMA-IR) were assessed.

Results: There were no differences in age and gestational age between the GDM group and the control group ( $\mathrm{p}=0.058$ and $\mathrm{p}=0.820$, respectively). Body mass index (BMI) at 24 to 28 weeks of gestation was higher in the GDM group ( $<0.001)$. The serum concentrations of RBP-4, chemerin, and visfatin did not demonstrate significant differences between the GDM and control groups $(p=0.871, p=0.100$, and $p=0.886$, respectively). Significant differences in serum level of insulin and HOMA-IR were found between the GDM and control groups (14.94 vs 9.87, $\mathrm{p}<0.001$ and 3.73 vs 1.77, $\mathrm{p}<0.001$, respectively). Correlation analyses of chemerin, RBP-4, visfatin, insulin, and HOMA-IR in both groups revealed a weak degree of positive correlation between RBP-4 and chemerin (Spearman $r=0.251, p=0.026$ ) and a strong positive correlation between maternal insulin and HOMA (Spearman $\mathrm{r}=0.868, \mathrm{p}<0.001$ ).

Conclusion: No differences were found in serum chemerin, RBP-4, and visfatin between pregnant women with GDM and healthy pregnant women. Further prospective studies will be essential to elucidate the contribution of adipokines to GDM and the positive correlation between maternal RBP-4 and chemerin. (J Turk Ger Gynecol Assoc 2016; 17: 186-90)
\end{abstract}

Keywords: Chemerin, retinol binding protein-4, visfatin, gestational diabetes

Received: 13 July, 2016 Accepted: 15 October, 2016

\section{Introduction}

Important alterations in maternal metabolism and increased insulin resistance coincide with the progressive accumulation of adiposity during the course of normal pregnancy. Insulin resistance shows an increase in the late second trimester to levels that are observed in type 2 diabetes mellitus (T2DM) (1). Most pregnant women remain normoglycemic due to adequate beta-cell compensation for this higher insulin secretion. When the beta-cell compensation for insulin resistance and hepatic glucose production is inadequate, gestational diabetes mellitus (GDM) ultimately develops (1). Moreover, $10 \%$ to $50 \%$ of GDM cases are reported to develop T2DM in the postpartum period (2).

Gestational diabetes mellitus is defined as a condition of carbohydrate intolerance with onset or first recognition during pregnancy (3). The American Diabetes Association states that the incidence of GDM is between $1 \%$ and $14 \%$, and it complicates almost $7 \%$ of pregnancies (4). GDM carries numerous risks for mothers, fetuses, and even offspring. GDM causes vascular and obstetric complications, including diabetic nephropathy, retinopathy, macrosomia, increased operative deliveries, and unexplained fetal demise. Neonatal complications, such as hypoglycemia, hypocalcemia, jaundice, respiratory distress syndrome, and cardiomyopathy, are also more prevalent. Offspring born to women with diabetes have a $1 \%$ to $3 \%$ risk of cardiovascular and metabolic disorders $(2,5)$. Maternal adiposity is an important, modifiable risk factor for the development of GDM (6). Adipose tissue is not only involved in energy storage, but also functions as an active endocrine organ (7). Recent evidence supports the crucial roles of specific hormones and adipokines (i.e., cytokines) secreted by adipose tissue. Among those identified to date are adiponectin, leptin, resistin, tumor necrosis factor alpha (TNF-alpha), progranulin, omentin, chemerin, retinol binding protein-4 (RBP-4), and visfatin.

Although adipokine chemerin has conventionally been associated with regulation of adaptive and innate immunity, it also induces insulin resistance and impairs glucose tolerance. Chemerin is a novel adipokine that is secreted by various tissues, especially white adipose tissue; it regulates insulin sensitivity in adipocytes and skeletal muscle $(8,9)$. Increased 
levels of chemerin are detected in obesity and are associated with multiple components of metabolic syndromes, including body mass index (BMI), triglycerides, high density cholesterol, hypertension, inflammation, and markers of liver pathology (10). Chemerin is also expressed in human placenta (11). Serum levels of chemerin correlate significantly with systemic markers of inflammation, such as TNF-alpha, interleukin 6, and C-reactive protein (12). Overall, these findings suggest that chemerin levels are related to adiposity and glucose metabolism. They also represent a possible link between obesity and the development of GDM.

Retinol binding protein-4 is a blood carrier protein for retinol that is synthesized in hepatocytes and adipocytes (13). Increased levels of RBP-4 have been demonstrated in several metabolic conditions, including obesity, insulin resistance, polycystic ovary syndrome, and cardiovascular disease (14). Also, RBP-4 is believed to induce expression of enzymes involved in gluconeogenesis in hepatocytes and to impair insulin signaling pathways in skeletal muscle (15).

Visfatin is predominantly secreted by visceral tissue; however, it is also found in skeletal muscle, liver, bone marrow, lymphocytes, and placenta (16). Visfatin promotes adipogenesis and exerts insulin-mimetic effects (17). It also upregulates production of proinflammatory cytokines by monocytes (18). Circulating levels of visfatin are increased in patients with type 1 and 2 DM and obesity $(13,19)$. However, the association of visfatin with GDM is still unclear (20).

Only a few adipokines have been investigated with respect to their involvement in GDM. Evidence in the existing literature does not support clear roles of chemerin, RBP-4, and visfatin in the prediction of GDM. In this study, we aimed to investigate the association of maternal serum levels of chemerin, RBP-4, visfatin, and insulin with GDM. Therefore, we performed a prospective cross-sectional study in pregnant women with GDM and with normal glucose tolerance.

\section{Material and Methods}

\section{Study population}

This cross-sectional study was conducted in the Obstetrics and Gynecology Department of Hitit University between March 2015 and September 2015. The ethics committee of Ankara Numune Hospital approved the study, which was in accordance with the Declaration of Helsinki, 2013 Brazil version (20796219-724.131). All participants gave written informed consent for the study. The patients were 18 to 35 years of age and had singleton pregnancies. Pregnant women who presented themselves to our obstetrics department were screened between 24 and 28 weeks of gestation for GDM according to the recommendations of the American College of Obstetricians and Gynecologists (ACOG) (24). Briefly, all pregnant women in the low risk group were evaluated with a 50-g glucose challenge test (GCT). Women with serum glucose $\geq 140 \mathrm{mg} / \mathrm{dL}$ at $1 \mathrm{~h}$ after GCT were subjected to a 100-g oral glucose tolerance test (OGTT). Serum glucose concentrations were measured at $0,1,2$, and $3 \mathrm{~h}$ after glucose ingestion. The diagnosis of GDM was based on the criteria of Carpenter and Couston, in which, after a 100-g oral glucose load, 2 or more of the following plasma values must be obtained: fasting $\geq 95 \mathrm{mg} / \mathrm{dL}, 1 \mathrm{~h} \geq 180 \mathrm{mg} / \mathrm{dL}, 2 \mathrm{~h} \geq 155 \mathrm{mg} / \mathrm{d} \mathrm{L}$, and $3 \mathrm{~h} \geq 140 \mathrm{mg} / \mathrm{dL}$ (21). The estimation of pregnancy duration was based on routine ultrasonographic examination performed in the first trimester. BMI was calculated using pregnancy weight and height, which were recorded at the time of blood sampling. The exclusion criteria were as follows: (1) smoking, (2) a history of diabetes mellitus and/or GDM, (3) a history of chronic disease, (4) a history of congenital malformation, (5) a family history of DM. A total of 158 pregnant women met the inclusion criteria and were divided into two groups: 76 were in the GDM group, and 82 were in the control group. The demographic characteristics and biochemical parameters of the study population, including age, BMI, and gestational age, were recorded in the second trimester.

\section{Assays}

Blood samples for adipokines and insulin were obtained from the antecubital vein after overnight fasting between 8:00 A.M. and 10:00 A.M. The samples for adipokines and insulin were centrifuged ( $1500 \mathrm{~g}$ for $25 \mathrm{~min}$ ), and the serum was immediately stored at $-80{ }^{\circ} \mathrm{C}$ until analysis. Serum glucose was determined daily using the glucose hexokinase method (Siemens Healthcare Diagnostic Limited; Camberley, UK). The serum chemerin concentration was measured by the enzyme-linked immunosorbent assay (ELISA) method (Biovendor, Biovendor-Laboratomi Medicina; Brno, Czech Republic). Serum RBP-4 concentration was determined by ELISA (Immundiagnostik, Immundiagnostik AG; Bensheim, Germany). Serum visfatin level was also determined by ELISA (Cusabio, Cusabio Biotech Co. Ltd.; Hubei, China). Serum insulin concentration was measured by chemiluminescence assay (Advia Centaur, Siemens Medical Solutions Diagnostics; Tarrytown, USA). Homeostasis model assessment-insulin resistance (HOMA-IR) was calculated using the following formula: Plasma glucose $(\mathrm{mg} / \mathrm{dL}) \times$ fasting plasma insulin (IUmg/L) in the fasting state divided by 405 (22).

\section{Statistical analysis}

Data were analyzed using SPSS (Statistical Package for the Social Sciences) software version 21 (SPSS Inc.; Chicago, IL, USA). Continuous variables were first evaluated for normality of statistical distribution by the Shapiro-Wilk test. As the continuous variables were not normally distributed, a non-parametric method (Mann-Whitney U test) was used to perform the statistical analysis. Descriptive statistics were expressed as the median (minimum-maximum) and number (percentage \%). Spearman correlation tests were used to determine the correlations of continuous variables. $\mathrm{P}$ values $<0.05$ were considered to be significant in all comparisons.

\section{Results}

The maternal demographic characteristics and biochemical parameters of the study participants are summarized in Table 1; all continuous variables are given as the median (minimummaximum). There were no differences in age and gestational 
Table 1. Comparison of maternal demographic characteristics and biochemical parameters in the gestational diabetes mellitus (GDM) and control groups

\begin{tabular}{|c|c|c|c|}
\hline & GDM group & Control group & $\mathbf{p}$ \\
\hline Number & $76(48.1 \%)$ & $82(51.9 \%)$ & \\
\hline Maternal age (years) & $29(19-35)$ & $26(18-35)$ & 0.058 \\
\hline $\begin{array}{l}\text { Gestational age } \\
\text { (weeks) }\end{array}$ & $26.5(24-28)$ & $25(24-28)$ & 0.820 \\
\hline BMI $\left(\mathrm{kg} / \mathrm{m}^{2}\right)$ & $\begin{array}{c}33.25 \\
(22.80-52.20)\end{array}$ & $\begin{array}{c}26.43 \\
(19.10-47.00)\end{array}$ & $0.000 *$ \\
\hline Chemerin (ng/mL) & $3.64(2.37-7.43)$ & 3.47 (2.19-4.63) & 0.100 \\
\hline RBP-4 (mg/mL) & $\begin{array}{c}15.29 \\
(10.08-19.63)\end{array}$ & $\begin{array}{c}14.91 \\
(10.64-31.16)\end{array}$ & 0.871 \\
\hline Visfatin (ng/mL) & $0.07(0.03-0.44)$ & $0.07(0.03-0.47)$ & 0.886 \\
\hline Insulin (mIU/mL) & $14.94(1.39-32.26)$ & 9.87 (3.53-23.98) & $0.000 *$ \\
\hline HOMA-IR & $3.73(0.33-8.40)$ & $1.77(0.60-4.59)$ & $0.000 *$ \\
\hline \multicolumn{4}{|c|}{$\begin{array}{l}\text { BMI: body mass index; RBP-4: retinol binding protein-4; HOMA-IR: homeostasis model } \\
\text { assessment of insulin resistance } \\
\text { "p values indicate statistical significance }(\mathrm{p}<0.05) . \\
\text { Values are shown as median (minimum-maximum). }\end{array}$} \\
\hline
\end{tabular}

Table 2. Correlation analyses of data

\begin{tabular}{|l|c|c|c|c|c|}
\hline & & Chemerin & Visfatin & Insulin & HOMA-IR \\
\hline \multirow{2}{*}{ RBP-4 } & $\mathrm{r}$ & 0.251 & -0.071 & -0.018 & 0.021 \\
\cline { 2 - 6 } & $\mathrm{p}$ & $0.026^{*}$ & 0.534 & 0.873 & 0.854 \\
\hline \multirow{3}{*}{ Chemerin } & $\mathrm{r}$ & & 0.192 & 0.196 & 0.161 \\
\cline { 2 - 6 } & $\mathrm{p}$ & & 0.090 & 0.083 & 0.155 \\
\hline \multirow{3}{*}{ Visfatin } & $\mathrm{r}$ & & & 0.143 & 0.071 \\
\cline { 2 - 6 } & $\mathrm{p}$ & & & 0.207 & 0.535 \\
\hline Insulin & $\mathrm{r}$ & & & & 0.868 \\
\cline { 2 - 6 } & $\mathrm{p}$ & & & & $0.000^{*}$ \\
\hline
\end{tabular}

RBP-4: retinol binding protein-4; HOMA-IR: homeostasis model assessment of insulin resistance

*p values indicate statistical significance $(\mathrm{p}<0.05)$.

age at the time of the study between the GDM group and the control group ( $p=0.058$ and $p=0.820$, respectively). However, the BMI of the GDM group was higher than that of the control group $(\mathrm{p}<0.001)$. The serum concentrations of RBP-4, chemerin, and visfatin did not demonstrate significant differences between the groups $(p=0.871, p=0.100$, and $p=0.886$, respectively). As expected, there were significant differences in serum levels of insulin and HOMA between the GDM and control groups [14.94 (1.39-32.26) vs $9.87(3.53-23.98, \mathrm{p}<0.001$ and $3.73(0.33-8.43)$ vs $1.77(0.6-4.59), \mathrm{p}<0.001$, respectively]. The insulin levels and HOMA of the GDM group were significantly higher than those of the control group. Correlation analyses of chemerin, RBP-4, visfatin, insulin, and HOMA-IR in both groups revealed a weak degree of positive correlation between maternal RBP-4 and chemerin (Spearman $\mathrm{r}=0.251, \mathrm{p}=0.026$ ) and a strong positive correlation between maternal insulin and HOMA (Spearman $\mathrm{r}=0.868, \mathrm{p}<0.001$ ), as shown in Table 2 .

\section{Discussion}

Various adipokines contribute to diabetogenic resistance to insulin, especially during the last half of pregnancy. This study mainly focused on potential alterations of specific adipokine concentrations (chemerin, RBP-4, and visfatin) in pregnant women with GDM. Here, we demonstrate that there were no significant differences in these adipokines between pregnant women with GDM and healthy pregnant women in the second trimester. However, the women with GDM were more likely to be overweight compared to matched healthy controls.

Chemerin has been proposed to be an insulin-sensitizing adipokine; its secretion has been demonstrated to increase, presumably as a compensatory mechanism, in insulin-resistant subjects (11). However, our results demonstrate the exact opposite. Various studies have evaluated the correlation of chemerin with clinical parameters in pregnant women. To date, no study has precisely determined the predictive value of chemerin concentration for risk of GDM. A recent study from Germany reported that circulating levels of chemerin did not show a significant difference between pregnant women with GDM and healthy pregnant controls (1). Garces et al. (23) found no significant association of insulin and HOMA levels with chemerin, as did other independent studies $(11,24,25)$. We found similar results for chemerin in pregnant women with GDM; however, other studies presented an association of chemerin with insulin resistance in pregnancy $(26,27)$. Overall, it appears that chemerin may not be useful in predicting GDM. As previously outlined, inconsistent data has been published concerning the association of RBP-4 with GDM. Some, but not all, studies have proposed that RBP-4 is positively and independently correlated with insulin resistance (28). Many studies demonstrated increased RBP-4 concentrations in $\operatorname{GDM}(14,29)$, while others did not show a relationship of chemerin with GDM or normal glucose tolerance, as in our study $(30,31)$. One study even reported that RBP-4 concentration was decreased in GDM (26). A study was conducted by Wójcik et al. (13) concerning the relationship between adipose tissue hormones and GDM. This findings of this study conflicted with those in previous reports.

In the present study, we also found that the visfatin levels of pregnant women with and without GDM were not significantly different. Current evidence regarding the association of visfatin concentration with GDM is contradictory. Some studies demonstrated increased serum levels of visfatin in women with GDM $(11,32,33)$, while others reported that visfatin concentrations were significantly lower in women with $\operatorname{GDM}(34,35)$. Contrary to our study, Lewandowski et al. (32) and Akturk et al. (35) reported a significant correlation between visfatin and HOMA. It is presumed that this discrepancy may be related to differences in gestational duration at the time of sampling, the variety of diagnostic criteria for GDM, or even racial differences (36). Furthermore, Morgan et al. (37) suggested that visfatin may act locally as a paracrine/autocrine agent and not as a hormone. However, the exact mechanism remains unclear. In a study by Rezvan, no correlation of visfatin was observed with insulin or HOMA (38). 
Despite the high number of studies evaluating the role of adipokines in GDM in the existing literature, interpretation of the results is somewhat laborious for several reasons. First, the diagnostic criteria for GDM vary greatly. Second, the gestational age at the study times ranges from early first trimester to late third trimester. Third, diverse assay methods may also cause heterogenous results. Obesity is accompanied by altered secretion of adipokines from adipose tissue $(39,40)$. Adipokine levels are usually higher in obese women. Although we demonstrated that pregnant women with GDM had higher BMI values than healthy pregnant women, there were no differences in the levels of the studied adipokines between the two groups. This may be due to the regulation of various adipokines by pregnancy or to insufficient matching of control and GDM patients for BMI. Limitations in the present study that should be mentioned are the relatively small number of participants in each group and the assessment of maternal adipokines only in the second trimester. Furthermore, we did not analyze adipokines during the pre-pregnancy period, during the course of pregnancy, and in the postpartum period. Finally, power analysis was not performed due to the limited financial resources of this study, which was supported by the Scientific Research Unit of Hitit University.

In conclusion, despite these limitations, our study supports that serum chemerin, RBP-4, and visfatin levels in pregnant women with GDM do not differ from those of healthy pregnant women. We suggest that long-term observations of adipokines during pre-pregnancy, pregnancy, and postpartum periods would increase our understanding of the pathogenesis of GDM. Therefore, further prospective studies are essential to elucidate the contribution of adipokines to GDM and the positive correlation between maternal RBP-4 and chemerin.

Ethics Committee Approval: Ethics committee approval was received for this study from the ethics committee of Ankara Numune Hospital (20796219-724.131).

Informed Consent: Written informed consent was obtained from patients who participated in this study.

Peer-review: Externally peer-reviewed.

Author Contributions: Concept - Ü.G.; Design - Ü.G.; Supervision T.G.; Data Collection and/or Processing - Ü.G., F.K.K.; Analysis and/ or Interpretation - Ü.G., C.T.; Literature Search - Ü.G., C.T.; Writing Manuscript - Ü.G.; Other - T.G.

Acknowledgements: The authors would like to thank the staff at Hitit University for collecting the data and their assistance.

Conflict of Interest: No conflict of interest was declared by the authors.

Financial Disclosure: The authors declared that this study has received financial support from the Scientific Research Unit of Hitit University, Çorum, Turkey.

\section{References}

1. Fasshauer M, Blüher M, Stumvoll M. Adipokines in gestational diabetes. Lancet Diabetes Endocrinol 2014; 2: 488-99.

2. Uzun G, Oktem S. Visfatin and its effects. Turk Klinik Biyokimya Derg 2013; 11: 119-30.

3. Teede HJ, Harrison CL, Teh WT, Paul E, Allan CA. Gestational diabetes: Development of an early risk prediction tool to facilitate op- portunities for prevention. Aust N Zeal J Obstet Gynaecol 2011; 51: 499-504.

4. American Diabetes Association. Gestational diabetes mellitus (Position Statement). Am J Obstet Gynecol 1987; 156: 488-9.

5. American Diabetes Association. Gestational diabetes mellitus. Diabetes Care 2004; 27(Suppl 1): S88-90.

6. Ben-Haroush A, Yogev Y, Hod M. Epidemiology of gestational diabetes mellitus and its association with type 2 diabetes. Diabet Med 2004; 21: 103-13.

7. Ouchi N, Parker JL, Lugus JJ, Walsh K. Adipokines in inflammation and metabolic disease. Nat Rev Immunol 2011; 11: 85-97.

8. Roman AA, Parlee SD, Sinal CJ. Chemerin: a potential endocrine link between obesity and type 2 diabetes. Endocrine 2012; 42: 24351.

9. Sell H, Laurencikiene J, Taube A, Eckardt K, Cramer A, Horrighs A, et al. Chemerin is a novel adipocyte-derived factor inducing insulin resistance in primary human skeletal muscle cells. Diabetes 2009; 58: 2731-40.

10. Catalán V, Gómez-Ambrosi J, Rodríguez A, Ramírez B, Rotellar $\mathrm{F}$, Valentí $\mathrm{V}$, et al. Increased levels of chemerin and its receptor, chemokine-like receptor-1, in obesity are related to inflammation: tumor necrosis factor- $\alpha$ stimulates mRNA levels of chemerin in visceral adipocytes from obese patients. Surg Obes Relat Dis 2013; 9: 306-14.

11. van Poppel MN, Zeck W, Ulrich D, Schest EC, Hirschmugl B, Lang U, et al. Cord blood chemerin: differential effects of gestational diabetes mellitus and maternal obesity. Clin Endocrinol 2014; 80: 65-72.

12. Wittamer V, Bondue B, Guillabert A, Vassart G, Parmentier M, Communi D. Neutrophil-mediated maturation of chemerin: a link between innate andadaptive immunity. J Immunol 2005; 175: 487-93.

13. Wójcik M, Chmielewska-Kassassir M, Grzywnowicz K, Woźniak L, Cypryk $\mathrm{K}$. The relationship between adipose tissue-derived hormones and gestational diabetes mellitus (GDM). Endokryno Polska 2014; 65: 134-42.

14. Miehle K, Stepan H, Fasshauer M. Leptin, adiponectin and other adipokines in gestational diabetes mellitus and pre-eclampsia. Clin Endocrinol 2012; 76: 2-11.

15. Bergmann K, Sypniewska G. Diabetes as a complication of adipose tissue dysfunction. Is there a role for potential new biomarkers? Clin Chem Lab Med 2013; 51: 177-85.

16. Fukuhara A, Matsuda M, Nishizawa M, Segawa K, Tanaka M, Kishimoto K, et al. Visfatin: A protein secreted by visceral fat that mimics the effects of insulin. Science 2005; 307: 426-30.

17. Briana DD, Malamitsi-Puchner A. Reviews: Adipocytokines in normal and complicated pregnancies. Reprod Sci 2009; 16: 921-37.

18. Moschen AR, Kaser A, Enrich B, Mosheimer B, Theurl M, Niederegger $\mathrm{H}$, et al. Visfatin, an adipocytokine with proinflammatory and immunomodulating properties. J Immunol 2007; 178: 1748-58.

19. Lopez-Bermejo A, Chico-Julia B, Fernandez-Balsells M, Recasens M, Esteve E, Casamitjana R, et al. Serum visfatin increases with progressive $\beta$-cell deterioration. Diabetes 2006; 55: 2871-5.

20. Haider DG, Handisurya A, Storka A, Vojtassakova E, Luger A, Pacini $\mathrm{G}$, et al. Visfatin response to glucose is reduced in women with gestational diabetes mellitus. Diabetes Care 2007; 30: 1889-91.

21. ACOG Practice Bulletin. Clinical management guidelines for obstetrician-gynecologists Number 30, September 2001 (replaces Technical Bulletin Number 200, December 1994). Gestational diabetes. Obstet Gynecol 2001; 98: 525-38.

22. Matthews DR, Hosker JP, Rudenski AS, Naylor BA, Treacher DF, Turner RC. Homeostasis model assessment: insulin resistance and beta-cell function from fasting plasma glucose and insulin concentrations in man. Diabetologia 1985; 28: 412-9. 
23. Garces MF, Sanchez E, Ruíz-Parra AI, Rubio-Romero JA, AngelMüller E, Suarez MA, et al. Serum chemerin levels during normal human pregnancy. Peptides 2013; 42: 138-43.

24. Barker G, Lim R, Rice GE, Lappas M. Increased chemerin concentrations in fetuses of obese mothers and correlation with maternal insulin sensitivity. J Matern Fetal Neonatal Med 2012; 25: 2274-80.

25. Pfau D, Stepan H, Kratzsch J, Verlohren M, Verlohren HJ, Drynda $\mathrm{K}$, et al. Circulating levels of the adipokine chemerin in gestational diabetes mellitus. Horm Res Paediatr 2010; 74: 56-61.

26. Hare KJ, Bonde L, Svare JA, Randeva HS, Asmar M, Larsen S, et al. Short Report: Pathophysiology Decreased plasma chemerin levels in women with gestational diabetes mellitus. Diabet Med 2014; 31 : 936-40.

27. Li XM, Ji H, Li CJ, Wang PH, Yu P, Yu DM. Chemerin expression in Chinese pregnant women with and withoutgestational diabetes mellitus. Ann Endocrinol (Paris) 2015; 76: 19-24.

28. Kotnik P, Fischer-Posovszky P, Wabitsch M. RBP4: a controversial adipokine. Eur J Endocrinol 2011; 165: 703-11.

29. Huang QT, Huang Q, Luo W, Li F, Hang LL, Yu YH, et al. Circulating retinol-binding protein 4 levels in gestational diabetes mellitus: A meta-analysis of observational studies. Gynecol Endocrinol 2015; 31: 337-44.

30. Nanda S, Nikoletakis G, Markova D, Poon LC, Nicolaides KH. Maternal serum retinol-binding protein- 4 at 11-13 weeks' gestation in normal and pathological pregnancies. Metabolism 2013; 62: 814-9.

31. Abetew DF, Qiu C, Fida NG, Dishi M, Hevner K, Williams MA, et al. Association of retinol binding protein 4 with risk of gestational diabetes. Diabetes Res Clin Pract 2013; 99: 48-53.
32. Lewandowski KC, Stojanovic N, Press M, Tuck SM, Szosland K, Bienkiewicz M, et al. Elevated serum levels of visfatin in gestational diabetes: a comparative study across various degrees of glucose tolerance. Diabetologia 2007; 50: 1033-7.

33. Coskun A, Ozkaya M, Kiran G, Kilinc M, Arikan DC. Plasma visfatin levels in pregnant women with normal glucose tolerance, gestational diabetes and pre-gestational diabetes mellitus. J Matern Fetal Neonatal Med 2010; 23: 1014-8.

34. Chan TF, Chen YL, Lee CH, Chou FH, Wu LC, Jong SB, et al. Decreased plasma visfatin concentrations in women with gestational diabetes mellitus. J Soc Gynecol Investig 2006; 13: 364-7.

35. Akturk M, Altinova AE, Mert I, Buyukkagnici U, Sargin A, Arslan M, et al. Visfatin concentration is decreased in women with gestational diabetes mellitus in the third trimester. J Endocrinol Invest 2006; 31: 610-3.

36. Krzyzanowska K, Krugluger W, Mittermayer F, Rahman R, Haider D, Shnawa N, et al. Increased visfatin concentrations in women with gestational diabetes mellitus. Clin Sci (Lond) 2006; 110: 605-9.

37. Morgan SA, Bringolf JB, Seidel ER. Visfatin expression is elevated in normal human pregnancy. Peptides 2008; 29: 1382-9.

38. Rezvan N, Hosseinzadeh-Attar MJ, Masoudkabir F, Moini A, Janani L, Mazaherioun M. Serum visfatin concentrations in gestational diabetes mellitus and normal pregnancy. Arch Gynecol Obstet 2012; 285: $1257-62$.

39. Rasouli N, Kern PA. Adipocytokines and the metabolic complications of obesity. J Clin Endocrinol Metab 2008; 93: S64-73.

40. Galic S, Oakhill JS, Steinberg GR. Adipose tissue as an endocrine organ. Mol Cell Endocrinol 2010; 316: 129-39. 